\title{
Super-Resolution Persistent Scatterer Interferometry (SR-PSI) - PS densification through Capon based SAR reprocessing
}

Hao Zhanga, ${ }^{\mathrm{a}}$ and Paco López-Dekker ${ }^{\mathrm{b}}$

${ }^{a}$ State Key Laboratory of Information Engineering in Surveying, Mapping and Remote Sensing, Wuhan University, Wuhan 430079, China

${ }^{b}$ the Department of Geoscience and Remote Sensing, Delft University of Technology, 2628CN Delft, The Netherlands

\begin{abstract}
The Capon algorithm can be applied to reprocess SAR images, resulting in super-resolution super-resolution reconstructed scenes with lower sidelobe levels. Base on this idea, we have proposed a processing chain of Super-Resolution Persistent Scatterer Interferometry (SR-PSI), to increase PS density. In this paper, we review the main aspect of SR-PSI. We also propose a revised robust Capon algorithm. The result of real-life Sentinel-1 (S-1) data is shown.
\end{abstract}

\section{Super-Resolution Persistent Scat- terer Interferometry (SR-PSI)}

The SAR imaging can be cast as a spectral estimation problem $[1,2,3]$. Let $\left\{s_{n_{1}, n_{2}}\right\}, n_{1}=0,1, \ldots, N_{1}-1, n_{2}=$ $0,1, \ldots, N_{2}-1$ denote a 2 -D data matrix and $\left(\omega_{1}, \omega_{2}\right)$ be the interested frequency pair. We can model $s_{n_{1}, n_{2}}$ as

$$
s\left(n_{1}, n_{2}\right)=\sum \alpha_{\omega_{1}, \omega_{2}} \cdot e^{j\left(n_{1} \cdot \omega_{1}+n_{2} \cdot \omega_{2}\right)}+e\left(n_{1}, n_{2}\right),
$$

where $\alpha_{\omega_{1}, \omega_{2}}$ denotes the complex amplitude of a sinusoid with frequency $\left(\omega_{1}, \omega_{2}\right)$, and $e\left(n_{1}, n_{2}\right)$ represents the unmodeled noise and interference. The focusing process is to estimate $\alpha\left(\omega_{1}, \omega_{2}\right)$ from the 2-D data $s_{n_{1}, n_{2}}$. The most efficient method is the 2-D Fourier transform, or matchedfilter based focusing algorithm. Single Look Complex (SLC) images generated through this approach suffer from the limited resolution and relatively high sidelobe levels [1]. We can apply modern spectral-estimation methods to calculate $\alpha\left(\omega_{1}, \omega_{2}\right)$, resulting in improved resolution and suppressed sidelobe levels $[1,3]$. By integrating these algorithms into Persistent Scatterer Interferometry, we can increase the PS density [4]. Since the Capon algorithm seems promising in the context of PSI, here we only consider the Capon algorithm.

A detailed discussion of the proposed Super-Resolution PSI (SR-PSI) can be found in [4]. Compared to the traditional PSI, the main change of SR-PSI is the SuperResolution reprocessing and PSC selection method. The step of Super-Resolution reprocessing is to refocus the SAR images using the Capon algorithm. It includes spectral equalization, image chipping, Discrete Fourier Transform (DFT), the Capon processing, and chip-images mosaicking [3] We can apply a peak-detection based Persistent Scatterer Candidate (PSC) selection approach to the reprocessed images rather than the regular normalized amplitude dispersion approach [4].

\section{Revised robust Capon algorithm}

\subsection{Revised robust Capon algorithm}

The Capon estimator is designed so that the power of the filtered signal is minimized with the constraint that the gain of the filter remains one at the selected frequency [5]:

$$
\min _{\mathbf{h}} \mathbf{h}^{\mathrm{H}} \mathbf{R h} \text {, subject to } \mathbf{h}^{\mathrm{H}} \mathbf{a}=1,
$$

where $\mathbf{R}$ is the covariance matrix, $\mathbf{a}$ is the Fourier matrix, $\mathbf{h}$ is the filter to be constructed and $(\cdot)^{\mathrm{H}}$ denotes conjugate transpose operator. The solution of (2) is given by

$$
\mathbf{h}=\frac{\mathbf{R}^{-1} \mathbf{a}}{\mathbf{a}^{\mathrm{H}} \mathbf{R}^{-1} \mathbf{a}}
$$

In the application of standard Capon algorithm, $\mathbf{R}$ is usually replaced by the sample covariance matrix $\hat{\mathbf{R}}$,

$$
\hat{\mathbf{R}}=\frac{1}{N} \sum_{n=1}^{N} \mathbf{z}(n) \mathbf{z}^{(*)}(n),
$$

where $N$ is the number of snapshots and $\mathbf{z}(\mathbf{n})$ is the $n$-th snapshot (snapshots are defined as the sub-matrices of the data matrix). To make the calculation more robust, a fully diagonal loading (DL) approach is proposed by [6]:

$$
\tilde{\mathbf{R}}=\alpha \mathbf{I}+\beta \hat{\mathbf{R}},
$$

is used instead of $\hat{\mathbf{R}}$. The $\alpha$ and $\beta$ coefficients are determined by minimizing the Mean Square Error (MSE) of $\tilde{\mathbf{R}}$ with the constraints that $\alpha \geq 0$ and $\beta \geq 0$, and guaranteeing that $\tilde{\mathbf{R}}$ is a positive semidefinite matrix. The solution of $\alpha$ and $\beta$ is expressed as:

$$
\hat{\alpha}_{0}=\min \left[\hat{v} \frac{\hat{\rho}}{\|\hat{\mathbf{R}}-\hat{v} \mathbf{I}\|^{2}}, \hat{v}\right], \hat{\beta}_{0}=1-\frac{\hat{\alpha}_{0}}{\hat{v}},
$$

where

$$
\hat{\rho}=\frac{1}{N^{2}} \sum_{n=1}^{N}\|\mathbf{z}(n)\|^{4}-\frac{1}{N}\|\hat{\mathbf{R}}\|^{2}
$$


and

$$
\hat{v}=\operatorname{tr}(\hat{\mathbf{R}}) / M .
$$

Since $\alpha$ and $\beta$ can be calculated from the data, the method is parameter-free. The covariance matrix $\tilde{\mathbf{R}}$ can be estimated by combining (5) and (6).

We found that DL approaches tend to cause discontinuities between the adjacent chip images. To avoid this as far as possible, we should apply the DL approach only when necessary. We set the required condition as when the condition number is above a given threshold. Thus the covariance matrix is estimated by

$$
\overline{\mathbf{R}}=\left\{\begin{array}{l}
\hat{\mathbf{R}}, \text { if } \operatorname{cond}(\hat{\mathbf{R}})<C_{0} \\
\tilde{\mathbf{R}}, \text { otherwise }
\end{array}\right.
$$

\subsection{Its effect on PSC selection}

The benefits of the proposed robust approach are illustrated in Fig. 1. We can observe edge effect on the upper subplot while the lower image is seamless. The edge effect results in spurious peaks that are identified as additional PSCs, as for example within the rectangle near the uppler left corner. We also observe that some new PSCs are present in the middle of the plot, which may indicate the figure reprocessed by regular Capon algorithm is resolved better than the one reprocessed by the standard automatic DL-Capon algorithm.

\section{Experimental Results}

We applied the robust-Capon-based SR-PSI to a stack of interferometric Sentinel-1 images. Fig. 2 shows a detailed comparison of the deformation velocities of the PSs. As indicated by the optical image, the two lines of PSs in the right subplot can be interpreted as dihedrals formed by the piers and the roof. We can observe the PSs are separated on the Capon-based reprocessed image while they are mixed on the original image, which shows that the resolution is improved. Correspondingly, the PS density is increased, which may confirms the effectiveness of SR-PSI.

\section{$4 \quad$ Literature}

[1] Stuart R DeGraaf. SAR imaging via modern 2-D spectral estimation methods. IEEE Transactions on Image Processing, 7(5):729-761, 1998.

[2] Jian Li and Petre Stoica. An adaptive filtering approach to spectral estimation and SAR imaging. IEEE Transactions on Signal Processing, 44(6):1469-1484, 1996.

[3] Paco López-Dekker and Jordi J Mallorquí. Capon-and APES-based SAR processing: performance and practical considerations. IEEE transactions on geoscience and remote sensing, 48(5):2388-2402, 2010.

[4] H. Zhang and P. López-Dekker. Persistent scatterer densification through the application of capon- and apes-based sar reprocessing algorithms. IEEE Transactions on Geoscience and Remote Sensing, 57:75217533, 2019.
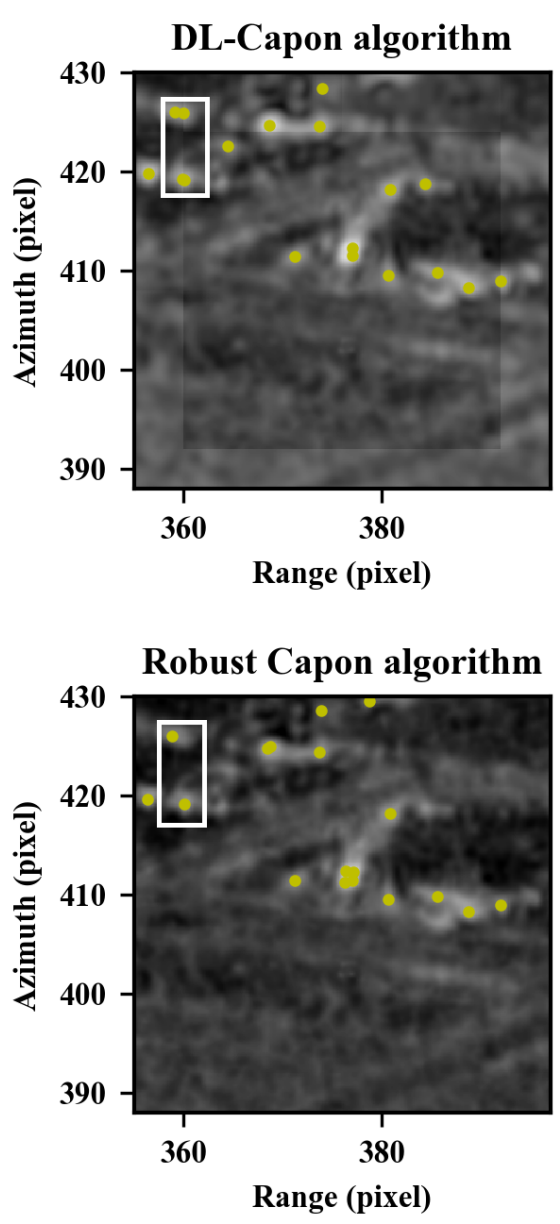

Figure 1 The upper subplot and the lower subplot present PSC selected from the images reprocessed by the automatic DL Capon algorithm and by our proposed algorithm, respectively.The background shows the squared mean intensity image.

[5] Jack Capon. High-resolution frequency-wavenumber spectrum analysis. Proceedings of the IEEE, 57(8):1408-1418, 1969.

[6] Lin Du, Jian Li, and Petre Stoica. Fully automatic computation of diagonal loading levels for robust adaptive beamforming. IEEE Transactions on Aerospace and Electronic Systems, 46(1), 2010. 

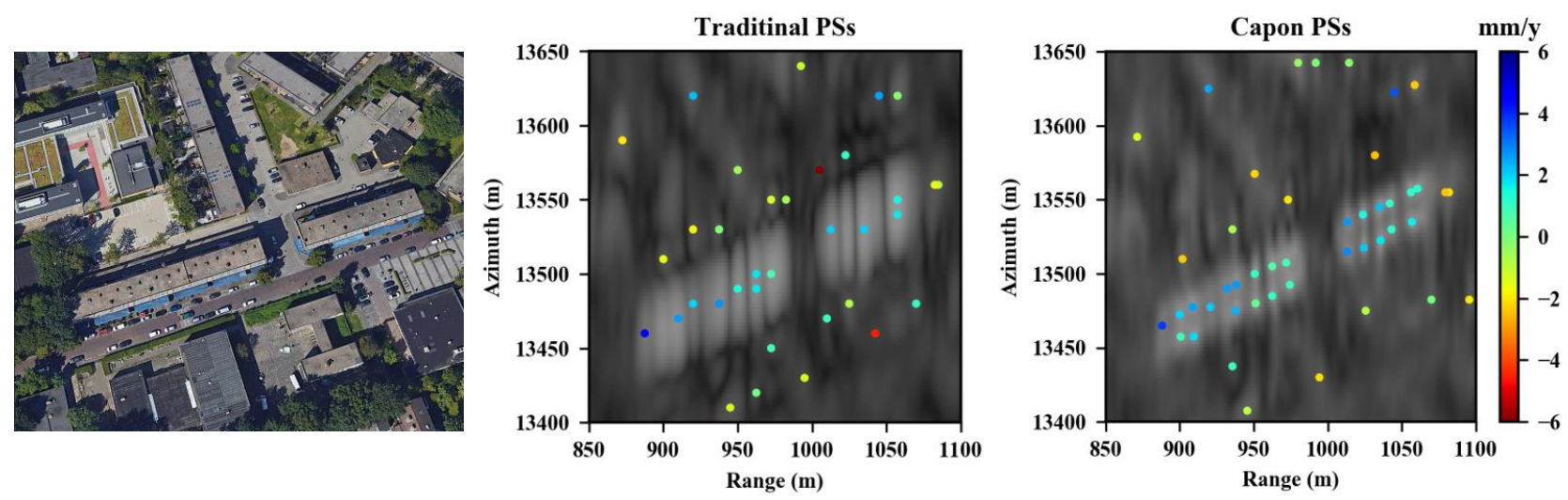

Figure 2 The left subplot shows the optical image of the area from the Google Earth. The middle subplot and the right subplot show the PSs selected from the original stack and from the Capon-based reprocessed images, respectively. 